\title{
BUBBLER TIP OF PYREX GLASS FOR DIFFICULT ABSORPTIONS
}

\author{
By Joseph R. Branham and Edward O. Sperling
}

\section{ABSTRACT}

This paper describes a tip made of Pyrex glass to be used in a gas-absorption pipette to divide the stream of gas into small bubbles in order to obtain a large surface of contact with liquid reagents. The tip is made by sealing a number of fine copper wires, radiating from a center in a definite pattern, between a cone formed on the end of a glass tube and a circular glass disk. After the glass parts are sealed together and ground to expose the ends of the wires, the wires are dissolved in warm nitric acid. Drawings which illustrate the method of making the tips are included. The tips are very sturdy and at moderate pressures furnish small bubbles of uniform size and distribution.

This paper describes a tip used for dividing a gas stream into bubbles for the washing of gases or for their absorption in liquid reagents. The tip was developed primarily for the absorption of oxygen in an alkaline solution of potassium pyrogallate in an Orsat apparatus. It is, of course, adaptable to other reagents and to other forms of apparatus. Each unit of the Orsat apparatus for which the tip was designed was made of Pyrex glass and sealed directly together to avoid rubber connections. ${ }^{1}$ The extent to which absorption is accelerated by dividing the gas stream passing through the reagent into small bubbles which offer a large surface of contact has been discussed in connection with two gas-absorption pipettes previously described. The gas entering the inlet tube of these pipettes is divided into small bubbles by passage through either a platinum disk drilled with several hundred orifices, $0.06 \mathrm{~mm}$ in diameter, ${ }^{2}$ or through a porous Jena glass thimble. ${ }^{3}$ Other types of porous or perforated disks have also been used to some extent, particularly in wash bottles.

The absorption pipette described in this paper is shown in figure 1. The dimensions of all drawings are given in millimeters. The front and top views of the assembled distributor tip are shown in figure 2 , $A$ and $B$, respectively. At the left, in figure 2, are shown crosssectional views of the parts $C, D$, and $E$, from which the tip is made and the top view of the copper spider, $F$, which is sealed between $C$ and $E$ at $D$. A thin copper disk, $G$, may be substituted for $F$, if desired. The spider, $F$, constructed of small copper wires, as is described later, or the thin copper disk, $G$, serve as temporary spacers which are later dissolved in nitric acid to leave channels of like shape in the glass. After $E$ is sealed to $C$ the outer ends of the wires forming

${ }^{1}$ For a discussion of errors caused by such connections, see, J. R. Branham, Errors in gas analysis arising from loss of gas by solution in rubber connections and stopcock lubricant, BS J. Research 12, 353 (1934) RP661.

3 Martin Shepherd, A gas analysis pipette for difficult absorptions. BS J. Research 4, 747 (1930) R P177.

'Martin Shepherd, Fish-Schurman Catalogue, p. 18. 
the spider are fully exposed to the original diameter of the wire by grinding the sides of the assembled tip, $A$.

The cross section of the graphite molds ${ }^{4}$ used to make $C$ and $E$ of figure 2 are shown in figure $3, A$ and $B$, respectively. The part of $A$ shown blackened in figure 3 is made of steel which has been heated and dipped into beeswax. When this mold is used, the upper section of $A$ is removed and slipped on a glass tube. The glass tube is then heated and thickened into a heavy-walled cone at its lower end.

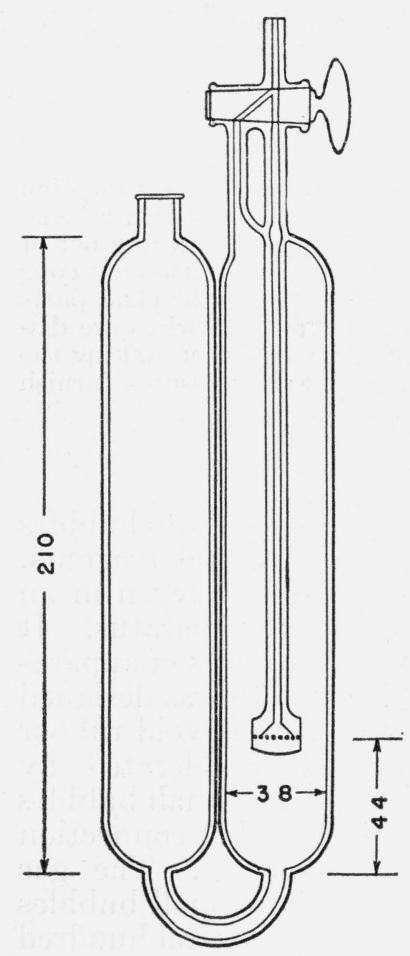

Figure 1.-A Pyrex absorption pipette and bubbler tip.

The hot softened glass is then inserted and pressed into the lower section of the mold, which is kept sufficiently hot to prevent the glass from cooling too rapidly. The glass disk, $E$ of figure 2, is formed at D, figure 3, in a similar manner, except that glass rod is used and a solid ball of glass is collected instead of the hollow cone. After the glass has cooled, it is file-scratched at $F$ and broken from the stem, $E$. The broken edge which results is ground nearly flat before the spider is sealed between the glass parts.

The copper spiders are made by winding a single length of copper wire on the hardened steel form, $A$, shown in figure 4 . This operation produces one spider at each end of the cylinder. The pegs on the side of the cylinder are used to secure one end of this wire at the start of the winding operation and the other end when the winding is finished. The windings pass nearly vertically up and down the sides of the cylinder and are guided across the top and bottom of the cylinder by the slots shown in $B$ of figure 4, so that each turn of wire crosses the axis of the cylinder. After winding is complete, that is, when 10 turns have been made and the ends of wire secured, two brass guides, each with a sliding steel plunger, are slipped over the ends of $A$. One of these guides is shown at $C$, figure 4. Sufficient pressure is then applied to the two plungers, by means of a vise, to mash the wires against a steel rod, the faces of which are flush with the bottom of the guiding slots. As a result of this operation the wires at the center of the spider become matted together sufficiently to permit handling the spider with forceps without its falling apart. Spiders have been made, as described, of copper wires of $0.20-, 0.16-, 0.13-$, and $0.10-\mathrm{mm}$ diameter. The spiders become increasingly fragile as the diameter of the wire decreases, and about one-half of the spiders made with $0.10-\mathrm{mm}$ wire fell apart when being handled. This fragility may have resulted from insufficient wire in the center of the spider to mat securely, or from a difference in the quality of the more finely drawn wire. The wires forming the spider are next cut with a sharp tool, for instance,

4 The authors are indebted to Harry Bailey of this Bureau for advice on design and for making the graphite and steel molds and forms used in this study. 
a safety-razor blade, ${ }^{5}$ at the outer edge of each slot (fig. $4, B$ ) and are then ready to be sealed into glass parts of the tip.

The sealing of the copper spider ${ }^{6}$ between $C$ and $E$ of figure 2 is done in two steps. (1) The spider is first placed between $C$ and $E$

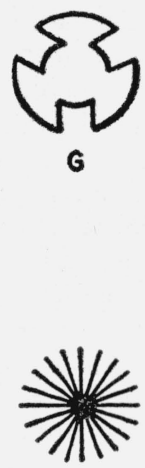

F

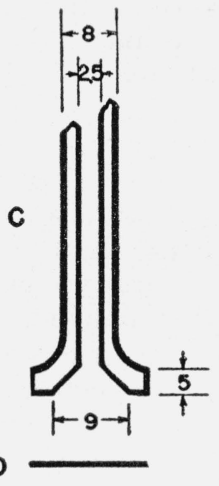

E

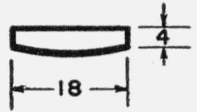

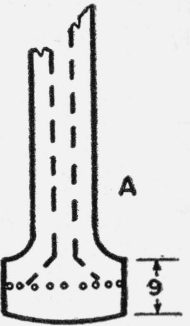

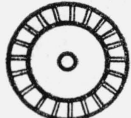

Figure 2.-Details of separate parts and assembled tip.

in the position shown in figure $5, C$ being supported in a hole bored in a transite block, $A$. The parts are then heated by a hand torch until the glass around the spider softens sufficiently to permit the spider to become partially imbedded in the glass so that its position will not
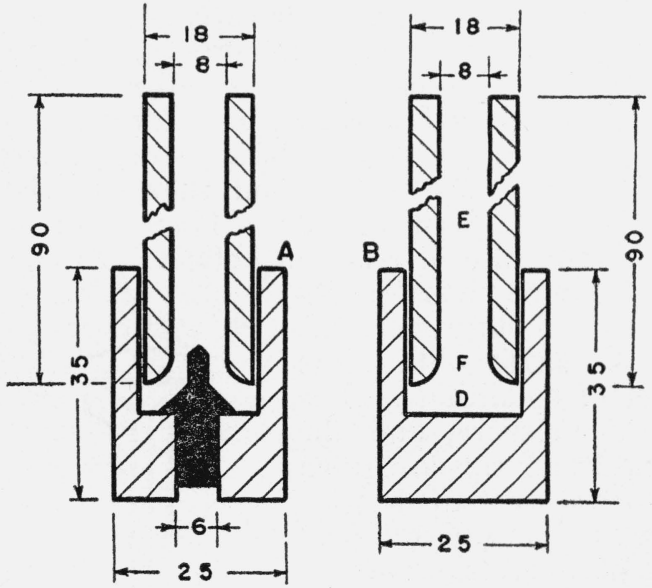

Figure 3.-Cross sections of graphite molds. change during the subsequent operations. The tip is then removed from the block and heated to a higher temperature in a blast lamp until the glass is soft enough to flow and completely fill the spaces between the wires when marvered. ${ }^{7}$

During these operations a thin layer of glass flows and covers the ends of the wires of the spider. ${ }^{8}$ This layer of glass is removed by inserting the tip into the chuck of a lathe and grinding it until the ends of the wires are exposed as uniform circles. The final

- This blade will cut the copper better if the angle between the sides of the cutting edge is increased to about 60 degrees by grinding it on a whetstone.

- Dipping the spider into a dilute solution of borax before this seal is made appears to be of no advantage but may help when $G$, figure 2, is substituted for $F$.

7 A glass-blowing term which denotes the application of pressure to a thoroughly heated piece of glass by rotating the glass while pressing it against a form of suitable material.

8 The diameter of the spider should be very slightly less than that of the glass parts, to prevent the wires from oxidizing or fusing. Extreme temperatures which might melt the wire and deform the circular channels should be avoided. 
operation is to dissolve the wires by immersing the tip in warm concentrated nitric acid, which leaves channels from which gases will issue as small bubbles.

During the earlier period of this study, several pipette tips similar to those described, except for the orifices, were made by using thin copper disks instead of the copper spiders which were developed later. One of these disks is shown at $G$ in figure 2. The disks are about $0.075 \mathrm{~mm}$ thick and slotted, as shown, to permit the glass

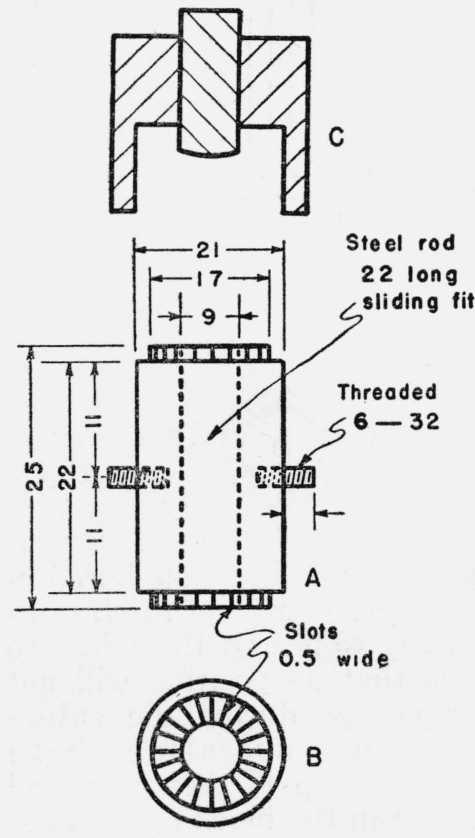

Figure 4.-Form used in making copper spiders. parts to be sealed together over the slotted areas. The bubble-making characteristics of these tips were about the same as those of the tips made with wire spiders. They were, however, somewhat more difficult to make, because excessive strains developed between the glass junctions and frequently caused the glass disk to separate from the cone when the tip was being ground to expose the edge of the disk. It was found in most cases that this breakage could be avoided by making several crisscross incisions at the center of the disk to relieve part of the shrinkage of the copper and by thoroughly annealing the tip after the copper disk had been sealed in place.

The size of the orifices in the pipette tip that should be selected for use for a given absorption will depend primarily on the pressure which can be used without danger of causing leakage of gas in the apparatus, and the rate at which it is desired to pass the gas through the reagent. These two factors determine the size of orifices which may be used and, therefore, the size of bubbles that will result in a given reagent. There are, of course, other variables, such as the rate of reaction between the gas and reagent, the physical solubility of "inert" gases in the reagent, and the dead space of the apparatus, which should be considered so as to obtain the best results under a given set of conditions. The relation between rate of flow, pressure, and the four sizes of orifices used in the Pyrex tips are given in figure 6 . These measurements were made with the tips immersed in water while air was bubbled through them. The depths of immersion of the tips below the surface of the water were subtracted from the observed total pressures. It was found advisable to increase the pressure of the gas slowly, until the liquid within the tip was discharged. This prevented the building up of excessive pressures which would cause a surge of relatively large bubbles of gas when the first portion of gas entered the liquid.

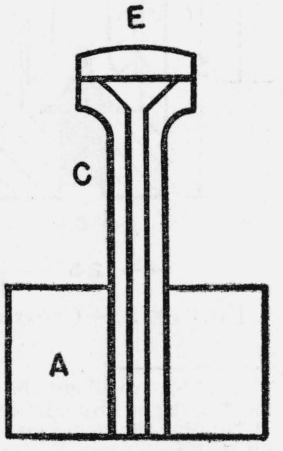

FIgURE 5.-Tip support used during initial sealing of copper spacers. 


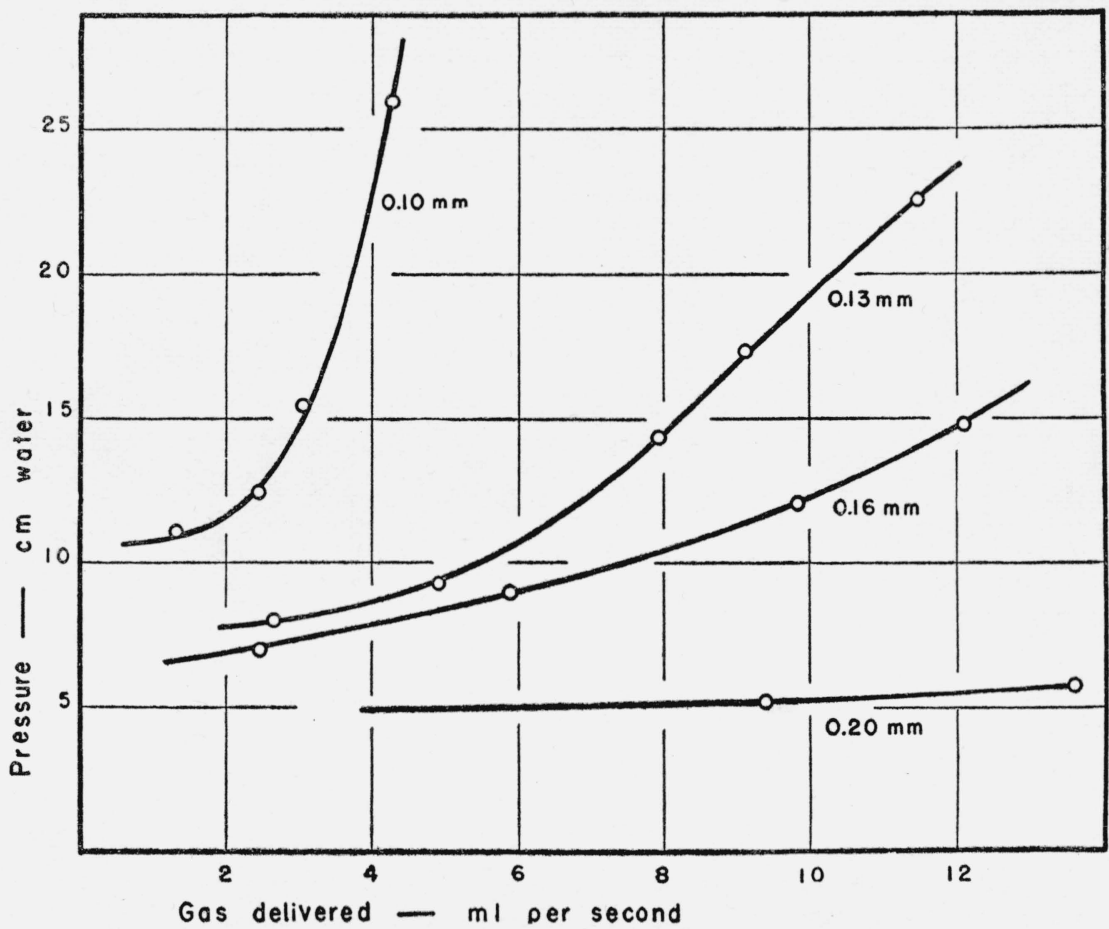

Figure 6.-Pressure-rate relations, air into water, for several sizes of orifices.

Gas-absorption pipettes equipped with tips of the kind described have been used successfully in this laboratory for the determination of oxygen by absorption in alkaline solutions of potassium pyrogallate. The tips are exceedingly sturdy and will withstand high pressures and rough treatment. The gas stream is divided by the tips into small bubbles of uniform size without subjecting the gas to excessive pressures.

Washington, March 1, 1939. 